\title{
El comer como instrumento. Alimentación e identidad entre los emigrantes vascos
}

\begin{abstract}
Las alubias de tu tierra y estas pochas tienen un diferente sentido del tiempo, tan viejo y oscuro como la sangre de esta morcilla o este pedazo de bárbaro chorizo casi disuelto por una cocción llena de parsimonia. Suenan dos botellas de vino tinto al destaponarse, Marqués de Murrieta, apuntas el nombre.
\end{abstract}

(M. Vázquez Montalbán. Galíndez).

Aquí los vascos se portan ciertamente de forma lamentable. Si disponen de una sopa de pimientos y vino en abundancia, se acuerdan de la religión como del viento ${ }^{1}$.

Puede parecer obvio el afirmar hoy día que la alimentación, los comportamientos alimentarios en general, se sitúan dentro del marco de las sociedades que los producen y recrean y, por tanto, dentro de sistemas socioculturales concretos desde los cuales vienen pautadas las características que los conforman. Utilizamos aquí el término comportamiento alimentario en el sentido que especifica Silvia Carrasco ${ }^{2}$, como la realidad referida, por un lado, a los alimentos y su manipulación, cargados de atributos culturales y, por otro, a los grupos humanos que participan y se organizan en sistemas alimentarios que se adaptan permanentemente para ser consistentes con su sistema sociocultural.

En tanto que producto social, los comportamientos alimentarios son interiorizados por los actores como elementos integrantes de un sistema sociocultural determinado, y que permiten las interrelaciones que hacen

1 Carta de un pastor vasco emigrante, desde las costas del Pacífico. Citada en Pierre LHANDE, La emigración vasca (Original en francés de 1910) (Donostia: Auñamendi, 1976).

2 Silvia CARRASCO, Antropologia i alimentació. Una proposta per a l'estudi de la cultura alimentària (Bellaterra: Universitat Autònoma de Barcelona, 1992). Cf. F. Xavier MEDINA, "Alimentación, dieta y comportamientos alimentarios en el contexto mediterráneo", F. Xavier MEDINA (ed.), La alimentación mediterránea. Historia, cultura, nutrición (Barcelona: Icaria, 1996). 
posible dentro del grupo la generación de vida en común; elementos que construyen y recrean la identidad grupal. En este sentido, la hipótesis aquí propuesta es la de plantear el comer como un instrumento que hace posible esta recreación de la identidad, y que permite a los individuos llevar a cabo su sistema de adscripciones dentro de una estructura de identificaciones concreta construida por los propios actores sociales ${ }^{3}$. Los comportamientos alimentarios son, asimismo, un instrumento explícito con que los emigrantes cuentan para recrear su identidad en la emigración. En las líneas que siguen llevaremos a cabo una aproximación a un caso concreto: el de la migración vasca, y, más concretamente, el de la migración vasca en la ciudad de Barcelona, a través del cual intentaremos desarrollar esta propuesta para un análisis de la construcción de la identidad a través de la alimentación.

\section{LA INMIGRACIÓN VASCA EN LA CIUDAD DE BARCELONA. APUNTES INTRODUCTORIOS}

Pese a no ser un modelo típico de migración económico-laboral tal y como se conoce habitualmente - especialmente en las grandes ciudades de un Estado como el español-, el grupo estudiado proporciona, sin embargo, la pauta de comportamiento de un grupo migrante establecido en un medio urbano en el cual ha reformulado su singularidad étnica, teniendo en cuenta tanto las características socioculturales que le son propias como el nuevo territorio en el que se encuentra asentado y que, en gran medida, ha convertido en suyo propio.

Un primer dato característico es su relativamente reducido volumen (que en poco excede de las ocho mil personas), siempre en comparación con otros grupos inmigrados residentes en Barcelona, como el andaluz, con más de ciento sesenta mil personas, el aragonés, con más de setenta mil, o el gallego, con cuarenta y cinco mil ${ }^{4}$. Otro factor de interés son sus períodos de llegada a la Ciudad Condal, con puntos álgidos en el lustro $1935-40$ y en la década 1971-80, en obvio contraste con los de individuos pertenecientes a grupos de una más clara tradición migratoria por motivos económico-laborales, con el punto máximo en la década 1961-70.

3 Cf. Mercedes FERNÁNDEZ-MARTORELl, "Usos del comer», Confluencias, II, 1 (1988), y Mercedes FERNÁNDEZ-MARTORELL, Creadores y vividores de ciudades. Ensayo de antropología urbana (Barcelona: EUB, 1996).

4 Cf. Padró d'babitants de la ciutat de Barcelona (Barcelona: Ajuntament de Barcelona, 1988). 
Podemos ver así que las épocas de llegada de los vascos a Barcelona no se corresponden con las de mayor afluencia migratoria por motivos laborales dentro del Estado español. Igualmente, y de los dos lapsos de tiempo señalados, el mayor contingente llegado a la Ciudad Condal desde Euskadi lo encontramos en el primero de ellos (1935-40); período que, precisamente, coincide con las fechas de la Guerra Civil, en que muchos vascos abandonaron su lugar de origen por causas directamente relacionadas con el conflicto bélico y encontraron en Barcelona un enclave privilegiado: por un lado, una ciudad con un tamaño suficientemente considerable que, asimismo, comenzaba a acusar ya las primeras oleadas migratorias; por otro, buenas posibilidades laborales en una economía de amplios antecedentes industriales; y un tercer factor importante en esos momentos de conflictividad: la relativa cercanía de la frontera francesa, ampliamente utilizada durante y después de la conflagración ${ }^{5}$.

Vemos aquí la primera de las causas de llegada a la capital catalana de algunos de los hoy residentes vascos (un 22,5\% del total de la población vasca residente). Pero existe aún otro período -el segundo en importancia- de afluencia de migración vasca hacia Barcelona: la década 1971-80. Aunque la fiebre de la emigración económica ha quedado ya atrás en buena medida en los años anteriores, es aun un período de fuerte inmigración en la ciudad con la llegada, por ejemplo, de un $14,8 \%$ de los hoy residentes andaluces. Podemos observar, sin embargo, que la emigración tradicional (económico-laboral) sufre una disminución importante: en el caso andaluz de un $20 \%$. A pesar de ello, es ahora cuando un nuevo contingente de personas decide su traslado a Barcelona desde Euskadi.

Observando el mapa urbano barcelonés, y en él las zonas en las que habita un mayor número de inmigrantes vascos, podremos percatarnos de que entre ellas se hallan algunas de las que ostentan una posición económica más elevada. En estas zonas vive un $42,5 \%$ de los miembros del colectivo estudiado y, curiosamente, buena parte de ellos llegaron a Barcelona entre 1971 y 1980 - junto a muchos otros situados en barrios de clase media- Los motivos laborales por los cuales estos "otros barceloneses" se encuentran en la ciudad no son los de una búsqueda indiscriminada de empleo sino que, en un gran porcentaje de los casos, es desde el mismo puesto de trabajo que ya se ocupaba en el lugar de origen como se accede, por cambio de destino, a la actual actividad en Barcelona. Este es el caso de varios ejecutivos de empresas, bancos o cajas de

5 F. Xavier MedinA, "La migració basca cap a Barcelona durant la Guerra Civil. Antropologia i Història a memòria del lehendakari Aguirre», Generació, 4 (1992). 
ahorros vascos que cuentan con filiales en la capital catalana, así como de otros técnicos y cargos medios que han sido requeridos y trasladados desde Euskadi.

Como ya habíamos expuesto anteriormente, a través de un estudio pormenorizado del grupo vasco podemos encontrar las pautas de comportamiento de un colectivo inmigrado residente en un marco urbano en el cual redefine sus peculiaridades étnicas. Aspectos como la alimentación pueden ser reveladores a este efecto.

\section{ALIMENTACIÓN Y MIGRACIÓN}

Como expresa el dicho catalán: "D'agafar culleres, n'hi ha de moltes maneres" ("Hay muchas formas de coger cucharas"). El comer se sitúa como instrumento dentro del campo de la identidad colectiva y se convierte, de este modo, en una de las fronteras simbólicas que hay que tener en cuenta entre grupos en contacto, convirtiéndose en una evidencia de la participación diferencial de los individuos en un marco sociocultural más amplio. Como expresa Igor de Garine ${ }^{6}$ : "no por casualidad la cocina figura en el primer plano en la panoplia de las reivindicaciones regionales".

Mediante los fenómenos migratorios la unidad espacial del grupo queda alterada, abocando a los actores a nuevas necesidades y formas de recreación de la identidad. De igual modo, los estilos alimentarios en una situación de emigración sufren un proceso de reorganización a todos los niveles; sin embargo, el sistema alimentario de los grupos inmigrados se somete a una dinámica que le es propia, incluso si no se diferencia del de la sociedad receptora en sus elementos estructurales más importantes ${ }^{7}$. En palabras de de Garine, parece ser que más allá de las influencias del medio "Se manifiesta en el ámbito de cada una de las culturas una preocupación por utilizar la alimentación para afirmar y fijar su cohesión interna y su heterogeneidad en relación a las culturas vecinas. Parece también que en el seno de cada cultura los alimentos y los platos son utilizados para explicitar distancias diferenciales entre los diferentes grupos operantes en la sociedad y entre diversas categorías de individuos" ${ }^{8}$.

En nuestro caso concreto, y en referencia al grupo vasco, la cultura de la alimentación no ha sido jamás desestimada. "El vasco tiene concien-

6 Igor DE GARINE, “Culture et nutrition", Communications, 31 (1979), p. 82.

7 Cf. Manuel CALVO, "Migration et alimentation", Social Science Information, 21, 3 (1982).

8 Igor DE GARINE, op. cit., p. 83. 
cia de que la alimentación es algo muy importante en la vida y, más aún, tiene conciencia de que en su país se come mejor que en ningún sitio, lo cual pudiera ser una nota más de etnocentrismo. Entre nosotros, a modo de broma, se suele decir que nuestro deporte nacional consiste en el banquete" ${ }^{9}$. Un informante nos decía:

... De eso no hay duda. A los vascos, si quieres tenerlos contentos en un sitio, que haya buena comida y buen vino. Si no, no hay nada que hacer. Por la comida se hace cualquier cosa, y por el vino es lo mismo. Porque en Euskadi se bebe mucho, ¿eh? En mi pueblo se dice que hay dos cosas de las que los vascos saben más que nadie: del tiempo que va a hacer y de vinos ${ }^{10}$.

\section{ALIMENTACIÓN E IDENTIDAD. LOS VASCOS EN BARCELONA}

Entre los vascos residentes en Barcelona los comportamientos alimentarios forman parte de la construcción y recreación de la identidad grupal, y existe consciencia, en este sentido, de que determinados alimentos integran un comportamiento alimentario grupal determinado:

No es que los platos sean diferentes... Bueno, sí... Es la manera de prepararlo... Pero sobre todo las cosas que ponemos... Es importante el pescado, sobre todo el bacalao y también la merluza, que son casi emblemáticos, aunque no sean sólo de nuestra cocina, porque aquí en Cataluña también se prepara el bacalao, por ejemplo a la "llauna", pero es diferente ${ }^{11}$.

No se trata, sin embargo, de señalar aquí un "régimen alimentario vasco" al cual poder recurrir como punto de referencia estático, sino de analizar cómo determinados elementos o factores integrantes del comportamiento alimentario del grupo estudiado - ya sean realmente tradicionales o introducidos de manera relativamente reciente ${ }^{12}$ - son indicados y reivindicados -utilizados como instrumentos- por los propios actores sociales como pertenecientes a una determinada estructura "grupal de identificaciones; como participantes en el proceso de definición sociocultural de la identidad. Como expresaba la misma informante anterior:

9 Gurutzi ARREGUI, "Alimentación y cultura", Maria-Àngels ROQUE (coord.), Encontre d'Antropologia i diversitat bispànica (Barcelona: Generalitat de Catalunya, Departament de Cultura, 1988), p. 307.

10 Varón. Guipuzcoano, 65 años.

11 Mujer. Vizcaína, 46 años.

12 Una informante nos explicaba: «Un día hablando con mi abuela sobre la comida, y eso, me decía: «Pero si las patatas vienen de América, y el bacalao lo pescan en el Norte, y las alubias son de no sé donde ¡Qué comía antes esta gente!». 
Los mismos nombres te lo dicen: merluza a la vasca, o bacalao a la vizcaína. ¡Son comidas vascas!

Cada grupo "posee un marco referencial que guía la elección de sus alimentos - algunos de ellos compartidos por otros grupos; otros son exclusivos- del cual el conjunto constituye un corpus más o menos estructurado de criterios que le pertenecen y que, asimismo, le confieren una particularidad diferencial, distintiva" ${ }^{13}$. La elección a nivel grupal de determinados alimentos -el bacalao o la merluza - como "representativos" de un comportamiento alimentario concreto, eleva a éstos al rango de identificadores socioculturales.

Sin embargo, el mantenimiento, de una tradición alimentaria específica por parte de los inmigrantes en sus lugares de residencia no es siempre fácil de llevar a cabo, dependiendo, entre otros factores, de las facilidades existentes para el aprovisionamiento de determinados ingredientes ${ }^{14}$. En el caso vasco, este problema reviste en sí mismo una escasa importancia, ya que buena parte de los elementos principales constituyentes de la cocina vasca son en principio de fácil acceso en Cataluña, y muy especialmente en Barcelona. Es interesante destacar en este sentido que, dándose la infraestructura de aprovisionamiento necesaria a su estilo alimentario, el grupo asegura en parte los medios para mantener su cultura, en lo que a alimentación se refiere.

En la ciudad de Barcelona y en su provincia existen determinados establecimientos especializados total o parcialmente en la venta de productos vascos (también navarros y riojanos) o relacionados con la cocina vasca que son de más difícil obtención o de producción más localizada —como pueden ser, por ejemplo, determinadas clases de vinos, quesos, etc. - y destinados casi exclusivamente a una clientela de estas procedencias, que debe de estar "asociada", obteniendo de este modo una información exclusiva y continuada sobre los productos a la venta, así como determinados descuentos sobre el precio de venta al público. Una de las formas de promoción de estos establecimientos es dirigirse por correo a aquellas personas de origen vasco, navarro o riojano, establecidas en Cataluña $-\mathrm{y}$, obviamente, en otros puntos de España-, ofreciendo sus servicios.

En buena parte de los casos, y a pesar de tener asegurado el aprovisionamiento de determinados productos, al formar éstos parte también del

13 Manuel CALvo, «La diversité des pratiques alimentaires dans le macro-espace social, Jean Peltre y Claude Thouvenot (eds.), Alimentation et régions (Nancy: Presses Universitaires de Nancy, 1989).

14 Cf. Anne RAulin, «Où s'approvisionne la Culture?», J. GUTWWIRTH y C. PÉTONNET (dirs.), Chemins de la ville. Enquêtes etbnologiques (Paris: Editions du CTHS, 1987). 
comportamiento alimentario de la sociedad receptora, la distinción a este nivel se da en la preparación, la combinación de los ingredientes, la cantidad, la presentación o la comensalidad. Este puede ser el caso, por ejemplo, del bacalao. En referencia a este elemento, compartido por ambas tradiciones culinarias, Vázquez Montalbán ${ }^{15}$ nos dice que también "Cataluña hizo de sus guisos de bacalao una de sus señas diferenciales en el contexto de las cocinas del Estado español, con logros casi tan excelentes como en la vasca, sin llegar a este plato sublime llamado bacalao al pil-pil. En Cataluña "Sobrevive el bacalao a la llauna o el bacalao con pasas $i$ buevos duros, pero no siempre con la materia prima a la altura del renombre de los platos".

La especificidad de ambas tradiciones culinarias se hace, de este modo, manifiesta, a pesar de la coincidencia de determinados elementos o ingredientes. Esta especificidad, sin embargo, se establece desde el interior del grupo, dependiendo del consenso de éste tanto la continuidad como los cambios que en dicho carácter puedan operarse. A partir de aquí, la comensalidad se convierte en uuno de los momentos más fuertemente socializados de todo sistema alimentario, dando el sentido de la homogeneidad del grupo, de su dinámican ${ }^{16}$. Fuera del ámbito familiar, y entre los residentes vascos en Barcelona, podemos establecer algunos espacios principales en los cuales la alimentación y la comensalidad grupal cobran su mayor significado. En las líneas que siguen revisaremos someramente dos de estos espacios: los restaurantes vascos y las sociedades gastronómicas.

La ciudad de Barcelona cuenta hoy en día con diversos - y algunos de ellos muy reputados - restaurantes especializados en cocina vasca. Distribuidos por buena parte de la geografía urbana, es posible constatar que, sin embargo, se da una cierta concentración de este tipo de locales en una zona concreta de la ciudad: el distrito de la dreta de l'Eixample y, más concretamente, en la calle de Sicilia, donde tan sólo entre los números 107 al 202 - manzanas consecutivas en esta calle- se encuentran cinco de los más renombrados restaurantes vascos de la ciudad, sin contar con algunos otros situados en las calles adyacentes, a pocos metros de los anteriores. Se da, de este modo, lo que podríamos llamar en parte una zona de especialización de este tipo de establecimientos dentro del marco urbano barcelonés, que se ha convertido en un importante lugar de reunión para los vascos residentes en la Ciudad Condal:

15 Manuel VÁzQuez MonTAlBÁN, L'art de menjar a Catalunya. Crònica de la resistència dels senyals d'identitat gastronòmica catalana (Barcelona: Edicions 62, 1977), pp. 146-147.

16 Manuel CALVO, «Migration et alimentation", Social Science Information, 21, 3 (1982), p. 408. 


\begin{abstract}
... Me acuerdo que cuando salíamos de estudiar, todos los que éramos vascos nos reuníamos en la Plaza Cataluña. Pero como a veces hubo algún problema (con las autoridades), nos fuimos a reunir después a la calle Sicilia, y allí nos íbamos todos a encontrar. Ten en cuenta que te estoy hablando de los años sesenta y tantos. Pero entonces había otro ambiente, y había algo por lo que luchar, y nosotros como vascos aquí nos sentíamos más unidos. [...] Yo, hace muchos años que sólo voy por allí de vez en cuando, y a lo que voy es a comer, al [cita el nombre de un restaurante], por ejemplo, que te sirven estupendamente. La otra noche fui y pedí un bacalao que estaba de miedo. Y además, te ponen una cantidad [...] Luego a veces el precio sí que resulta un poco caro, pero vale la pena, ¿eh? ${ }^{17}$
\end{abstract}

Los restaurantes de la calle de Sicilia se convirtieron ya desde la segunda mitad del franquismo en un concurrido lugar de reunión para los residentes vascos de Barcelona y hoy en día son aún un importante lugar de encuentro, de sociabilidad:

Me paso por ahí cuando nos juntamos unos cuantos, o cuando estoy sólo, sobre todo los fines de semana, que seguro que me encuentro a alguien.

En una línea diferente, aunque en íntima relación con los restaurantes, ha aparecido en los últimos años otro tipo de locales que, aunque también dedicados a la restauración, centran la mayor parte de su actividad - y su fama- en las especialidades y variedad de pinchos y tapas elaborados, la mayor parte, al estilo donostiarra. Es interesante a este respecto destacar que, tal como hemos visto en el caso anterior, con este tipo de locales ha comenzado a crearse también recientemente, entre 1994 y 1995, una nueva e incipiente zona de especialización, esta vez en el céntrico distrito de Ciutat Vella. Principalmente a raíz del éxito obtenido por el restaurante de la Euskal Etxea - casa vasca- de Barcelona, reinaugurada en esta zona en 1994, otras dos tabernas-restaurantes han abierto sus puertas, casi al mismo tiempo, a escasos metros del anterior. En ambos casos, los promotores de la iniciativa han sido personas ya relacionadas con la restauración vasca en Cataluña, dueños de otros restaurantes o bares, bien en Barcelona, bien en otras localidades cercanas.

Como observan Douglass y Bilbao ${ }^{18}$ para el caso de los hoteles vascos del Oeste de los Estados Unidos, los establecimientos mencionados ofrecen en parte al visitante vasco un ambiente y una comida que recrea los existentes $\longrightarrow$ los supuestamente existentes- en Euskadi, al tiempo

17 Varón. Vizcaíno, 54 años.

18 William A. Douglass y Jon BILBAO, Amerikanuak. Los vascos en el Nuevo Mundo (Original en inglés de 1975) (Lejona: Universidad del País Vasco-Euskal Herriko Unibersitatea, 1986). 
que ofrecen la posibilidad de entrar en contacto con otros individuos de su lugar de origen.

Hay que destacar a este respecto cómo, entre los grupos inmigrantes, determinados alimentos que consuetudinariamente pueden pertenecer a la tradición gastronómica colectiva en el lugar de residencia, no tienen porqué pertenecer a la del grupo en un sentido más amplio. Douglass y Bilbao ${ }^{19}$ nos ofrecen el ejemplo del ponche picón entre los vascoamericanos: "Una bebida predilecta en Europa a principios de siglo, hecha de licor de picón, granadina, coñac y soda [que] fue extraordinariamente popular entre los vascos del Oeste [de los Estados Unidos]. Actualmente, el ponche picón - Picon punch - se considera como una bebida típica vasca, aunque hoy en día resulta casi imposible comprar un ponche picón en el País Vascon.

Aunque en un terreno más restringido que bordea la línea de la privacidad, el caso de las llamadas sociedades gastronómicas $\longrightarrow$ txokos en Bizkaia- es también significativo para nuestro análisis. Como organizaciones informales, las sociedades gastronómicas son agrupaciones compuestas por una serie de miembros, regidas por una junta directiva con unos objetivos expuestos en sus estatutos - principalmente las actividades gastronómicas-, y cuentan siempre con un local, en propiedad o no, habilitado para los fines de la sociedad ${ }^{20}$. Estas "sociedades" -más de mil actualmente en Euskadi- se han convertido a lo largo de los años en uno de los reductos más importantes, tanto de la privacidad masculina -es ampliamente conocida la exclusión de las mujeres de las reuniones de las sociedades - como de la cocina vasca tradicional. En palabras del cocinero Juan Mari Arzak, "Lo que entendemos por cocina vasca tradicional ha permanecido prácticamente inalterable en el tiempo, transmitiéndose sus secretos por vía femenina en los fogones domésticos ${ }^{21}$ y por vía masculina en las sociedades gastronómicas ${ }^{22}$.

Vemos a través de esta declaración cómo se concede popularmente a los Txokos, en el imaginario colectivo, un papel de primordial importancia en la transmisión de una prácticamente inalterable herencia gastronó-

19 William A. Douglass y Jon BilbaO, op. cit., p. 463.

20 Cf. Eugenia RAMírez GOICOECHEA, De jóvenes y sus identidades. Socioantropologia de la etnicidad en Euskadi (Madrid: CIS, 1991), p. 272.

21 Una interesantísima y pormenorizada información etnográfica sobre la alimentación en el ámbito doméstico puede encontrarse en José Miguel DE BARANDIARÁN y Ander MANTEROLA (dirs.), Atlas etnográfico de Vasconia. La alimentación doméstica en Vasconia (Bilbao: Eusko Jaurlaritza-Etniker Euskalerria, 1990).

22 Juan María ARZAK, "Presentación", en P. A. IDROQUILIS, Gastronomía (Bilbao: Departamento de Comercio, Consumo y Turismo, Gobierno Vasco, 1992), p. 7. La cursiva es nuestra. 
mica vasca por vía masculina, en el mismo nivel que el ámbito del hogar - los fogones domésticos-, cuya transmisión pasa por la vía femenina. Con respecto a la mencionada exclusión de las mujeres, en un reportaje publicado en la prensa barcelonesa, se cita la declaración de un txokero:

Si entrasen mujeres esto se acabaría, habría líos, criticarían cómo cocinamos o lo que bebemos y se iría todo al carajo, como ya ha sucedido de hecho donde se las ha dejado entrar ${ }^{23}$.

Ramírez Goicoechea ${ }^{24}$ dice al respecto que este tipo de asociaciones se perfilan "Como una institución de la sociedad masculina que cristaliza en el encuentro de la sociedad urbana industrial [...] con un substrato tradicional que consagra la segregación entre los espacios masculino y femenino". Esta situación, sin embargo, ha ido sufriendo cambios en los últimos años, en los que se ha pasado de dejar entrar a las mujeres y demás familiares de los socios durante un día a la semana establecido de antemano - principalmente los fines de semana- a constituir algunas sociedades mixtas, en las cuales se ha aceptado la presencia de mujeres como socios de pleno derecho, e incluso a la creación de algunas sociedades formadas exclusivamente por mujeres.

En la ciudad de Barcelona existe hoy en día un pequeño número de sociedades gastronómicas, que cuentan con un reducido volumen de socios. Una de las más tradicionales y antiguas de las establecidas en la ciudad es Euskal Zaleak, situada en el casco viejo de la ciudad, relativamente cerca del Mercat del Born.

¡Hombre!, queríamos tener un txoko aquí. Muchos habíamos estado ya en otros antes de venir, y echábamos de menos las reuniones, las comidas en grupo, y eso. Pero también el hecho de no estar en casa ... No sé, es un sitio propio, ¿no? De los socios. Aunque últimamente, entre los unos y los otros, esto se pone de gente insoportable: que si los amigos de uno que vienen, que si el otro los trae a los otros. Y así va ${ }^{25}$.

Todo gira, en estas sociedades, en torno a la cocina. Sus miembros se reunen para hacer comidas o cenas en conjunto, y pertenecen a ella, junto a la mayoría de socios de origen vasco, algunos otros de distinta procedencia, principalmente catalanes.

Yo tenía amigos vascos, y me contaban a veces, de cómo funcionaban los Txokos en Euskadi, que sólo iban los maridos ... Todo, ¿no? Y [ ..]. Pues te hace tener un

23 Antoni BATISTA, "Lo que se cuece en Euskadi», La Vanguardia Magazine (Barcelona, 15 de noviembre de 1992), p. 18.

24 Eugenia Ramírez Goicoechea, op. cit., p. 287.

25 Varón. Vizcaíno, 68 años. 
poco de curiosidad, ¿no? Y luego, cuando me dijeron que abrían uno aquí, y que podía entrar [...] La verdad es que no me lo pensé mucho. [...] Además, ¿Tú sabes lo que es venir aquí, lo bien que se come? Yo porque sólo me defiendo, no tengo mano, pero [nombre de un socio del Txoko] te hace cada plato que... ${ }^{26}$

Las mujeres tienen acceso al Txoko, aunque solamente como acompañantes ("iQué remedio!n) y en aquellos días en los que se permite la entrada a personal ajeno a la sociedad. El espacio de sociabilidad masculina, de todas formas, es respetado, aunque más por las mujeres de una cierta edad que por las más jóvenes:

¡Éste! [refiriéndose a su marido] ¡Cualquiera lo tiene en casa! Pero por mí, mejor, ¿eh? Porque, ¿sabes qué te digo?, que prefiero que se vaya a la sociedad, porque por lo menos sé dónde se mete, y cuando llega la hora, iHale, a casa! ${ }^{27}$

¿Que no nos dejan entrar?, pues que no nos dejen. Ya haremos nosotras la nuestra. Además, si ahí no quedan más que los cuatro viejos [...] A mí dame el salir de copas, la marcha y tal [...] De todas formas, claro que está mal que no sean socias las mujeres. Estamos con lo de siempre, la vena machista. Pero, bueno, los jóvenes pasan más ¿no? Son los más viejos ${ }^{28}$.

A pesar de las diferencias que el tema suscita, los Txokos forman parte íntima del imaginario reivindicado como más tradicional del grupo vasco residente en Barcelona. Del mismo modo, la sociabilidad en un ambiente autorreconocido como grupal, y alrededor de unos platos que consuetudinariamente pertenecen a una tradición culinaria determinada, hace partícipes a los individuos del proceso de construcción y de recreación sociocultural de la identidad colectiva, dentro de una determinada estructura de identificaciones.

En cada uno de los ámbitos analizados, el comer es utilizado por los propios actores como un instrumento de identificación. En estas líneas hemos presentado esta posible propuesta para un análisis sociocultural de la identidad a través de los comportamientos alimentarios de los individuos. De este modo, la alimentación queda ubicada, fuera de toda duda, mucho más allá de las consideraciones biológicas y nutricionales, dentro del marco cultural de las relaciones sociales.

\author{
F. XAVIER MEDINA \\ Institut Català de la Mediterrània \\ d'Estudis i Cooperació. Barcelona
}

\footnotetext{
26 Varón. Barcelonés, 54 años.

27 Mujer. Guipuzcoana, 68 años.

28 Mujer. Barcelonesa (hija de vascos), 24 años.
} 
Los comportamientos alimentarios se construyen dentro del marco de las relaciones sociales entre los individuos. Este artículo propone, a través del ejemplo concreto de la inmigración vasca en la ciudad de Barcelona, la posibilidad de plantear la alimentación, el comer, como un fenómeno implicado en la construcción de la identidad grupal, dentro de una estructura de identificaciones específica. Esta hipótesis se ejemplifica a partir de la revisión de dos casos concretos: los restaurantes vascos y las sociedades gastronómicas en Barcelona.

Food habits are constructed within the context of social relations among individuals. The present article aims at showing, through the case of Basque immigration in Barcelona, how food -eating - can be a factor in building group identity within a specific structure or identifications. The merits of this hypothesis are grounded in the study of Basque restaurants as well as gastronomic societies in Barcelona. 